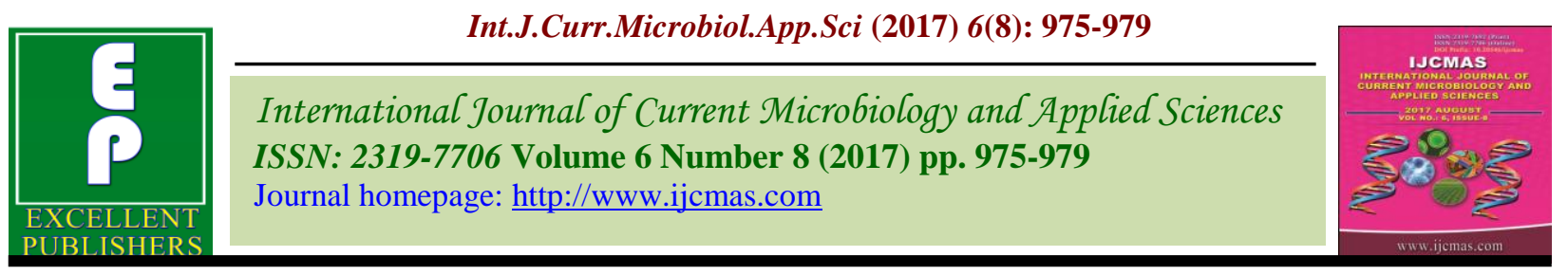

Original Research Article

https://doi.org/10.20546/ijcmas.2017.608.119

\title{
Laparoscopy Guided PCNL in a Stone Holding Previously Operated Ectopic Pelvic Kidney
}

\author{
Bhushan V. Dodia*, Abhay Mahajan, Martand Patil, Sandeep Bathe and Prashant Darakh
}

MGM Institute of Medical Science and Hospital, Aurangabad, India

*Corresponding author

\section{A B S T R A C T}

\begin{tabular}{|l|}
\hline K e y w o r d s \\
Laparoscopy \\
assisted PCNL, \\
PCNL, Pelvic \\
ectopic kidney, \\
Urolithiasis. \\
\hline Article Info \\
\hline $\begin{array}{l}\text { Accepted: } \\
\text { 14 June } 2017 \\
\text { Available Online: } \\
\text { 10 August } 2017\end{array}$ \\
\hline
\end{tabular}

\section{Introduction}

Urologists are much familiar with operating renal stones in normally lying well ascended retroperitoneal kidneys. However renal stones in anomalous pelvic dystopic kidney is a task bit difficult. Conventional PCNL cannot be easily done in these condition because of overlying bone and risk of injuring bowel and anomalous blood supply of low lying kidney. Such patient can be safely dealt with Laparoscopy guided PCNL without any major complications.

\section{History}

36 years old female patient presented with chief complaints of dull aching pain in right iliac region with repeated colics associated with nausea and vomiting. Her menstrual history was normal. Past history suggestive of open surgery done for renal stone on right side. Per abdominal examination showed scar mark of previous surgery in right iliac fossa.

On investigations, she was found to have normal hemogram and renal functional test and her ultrasonography findings showed $\mathrm{rt}$ sided ectopic malrotated kidney with pelvic calculus of $22 \mathrm{~mm}$ size with mild $\mathrm{HN}$ and $4 \mathrm{~cm}$ uterine subserosal fibroid.

IVP showed rt ectopic malrotated kidney with pelvic calculus and mild $\mathrm{HN}$ with good functional draining pattern (Figure 1).

Since pelvic ectopic kidneys are prone for anomalous blood supply and abnormal 
orientation $\mathrm{Ct}$ Angiogram of the patient was done, which showed anomalous blood supply of pelvic lying kidney with pelvis facing anteriorly and calyces posteriorly with a pelvic calculus.

DTPA Scan of the patient was done which showed non-obstructed drainage with 49\% renal function of right kidney. Looking at investigatory profile with ectopic pelvic kidney and pelvic calculus and past history of open surgery on same side for nephrolithiasis, patient was decided to be posted for laparoscopy assisted PCNL.

After anaesthetising the patient ureteric catheterisation was done under fluoroscopy guidance. Later patient was kept in supine position and laparoscopic $10 \mathrm{~mm}$ umbilical port was inserted with Veress needle technique, $5 \mathrm{~mm}$ ports were created in left iliac region.

With laparoscopy renal bulge was identified and overlying bowel loops were displaced with laparoscopy forceps. Under direct vision with a laparoscopy on a separate video monitor and under fluoroscopic guidance PCS puncture was taken and after confirming inside Pelvicalyceal system serial dilation was done with metal dilators and amplatz was laid (Figure 2).

Nephroscopy showed pelvic calculus which was broken with ISWL and fragments removed with forceps. Nephrostomy was kept at the end of procedure after deploying 4.5/16 DJ stent (Figure 3).

Post operatively patient had uneventful event. Nephrostomy was removed on day 2 and patient was discharged by day 5 . Patient had stent removal after 1 month. Patient had short stay compared to her previous surgery and had advantage of less post-operative pain with earlier recovery and no major complications.

\section{Results and Discussion}

Treatment of Renal stones has gone a drastic change in today's world ranging from open surgeries, SWL and Endoscopic Management where amalgamation with laparoscopy and robots have made the task more easier. In today's scenario open renal surgery accounts for only $2-5 \%$ of cases [3].

Urologist are much familiar with various endourological procedures like URS, PCNL, and also with advance procedures like Mini PERC, RIRS, Ultramini PERC in normally lying well ascended kidneys.

However performing such procedures in ectopic kidneys is a unique challenge to the urologist [3]. Although a simple ectopic kidney may seldom be responsible for symptoms but association with malrotation and anatomic variations increases risk for hydronephrosis, hematuria, infection and stone formation [4].

Symptomatic ectopic pelvic kidneys with renal lithiasis poses problem in management due to abnormal location, malrotation, anomalous blood supply and operative risk for intraperitoneal urinary extravasation causing ileus and proximity of bowel during renal access [1].

Wide no of Treatment options are available for renal lithiasis in pelvic kidneys like open surgery, ESWL, PCNL, Laparoscopy, RIRS [2].

Open surgery increases morbidity due to bowel manipulation, larger scar, increased pain and cosmetically acceptable.

SWL in pelvic kidneys has reported success rate of 54- $68 \%$ and is less effective due to surrounding bowel and bone, high insertion of ureter with accompanying impaired mobility significantly hampering clearance of stone 
fragments. RIRS has become a much popular technique nowadays but requires experienced surgeon and procedure itself sometimes becomes difficult in large stone burden and tortuous ureters.
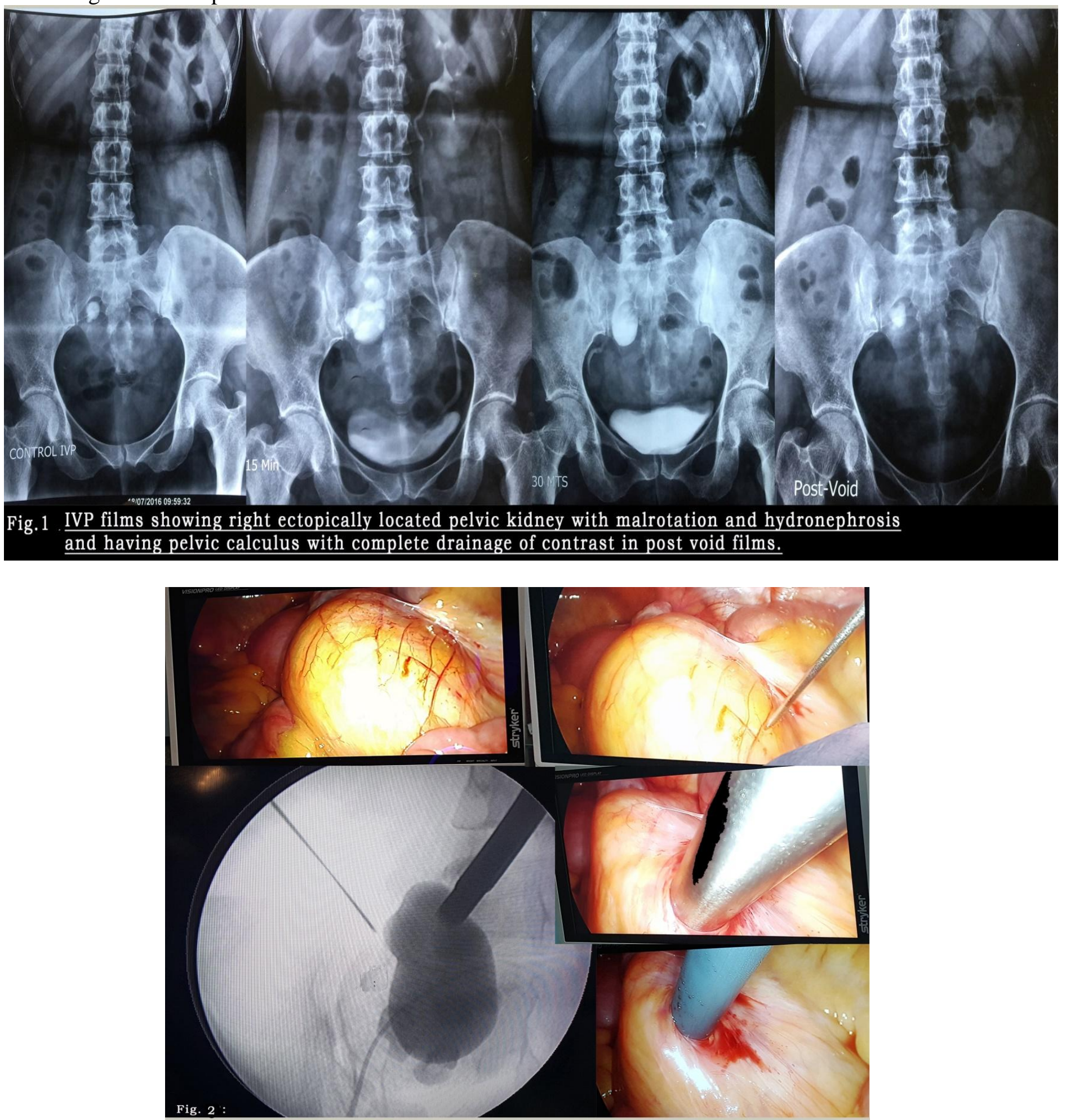

Picture showing PCNL puncture taken under laparoscopy guidance mobilising the overlying bowel with laparoscopic forceps and tract dilation under direct vision. 


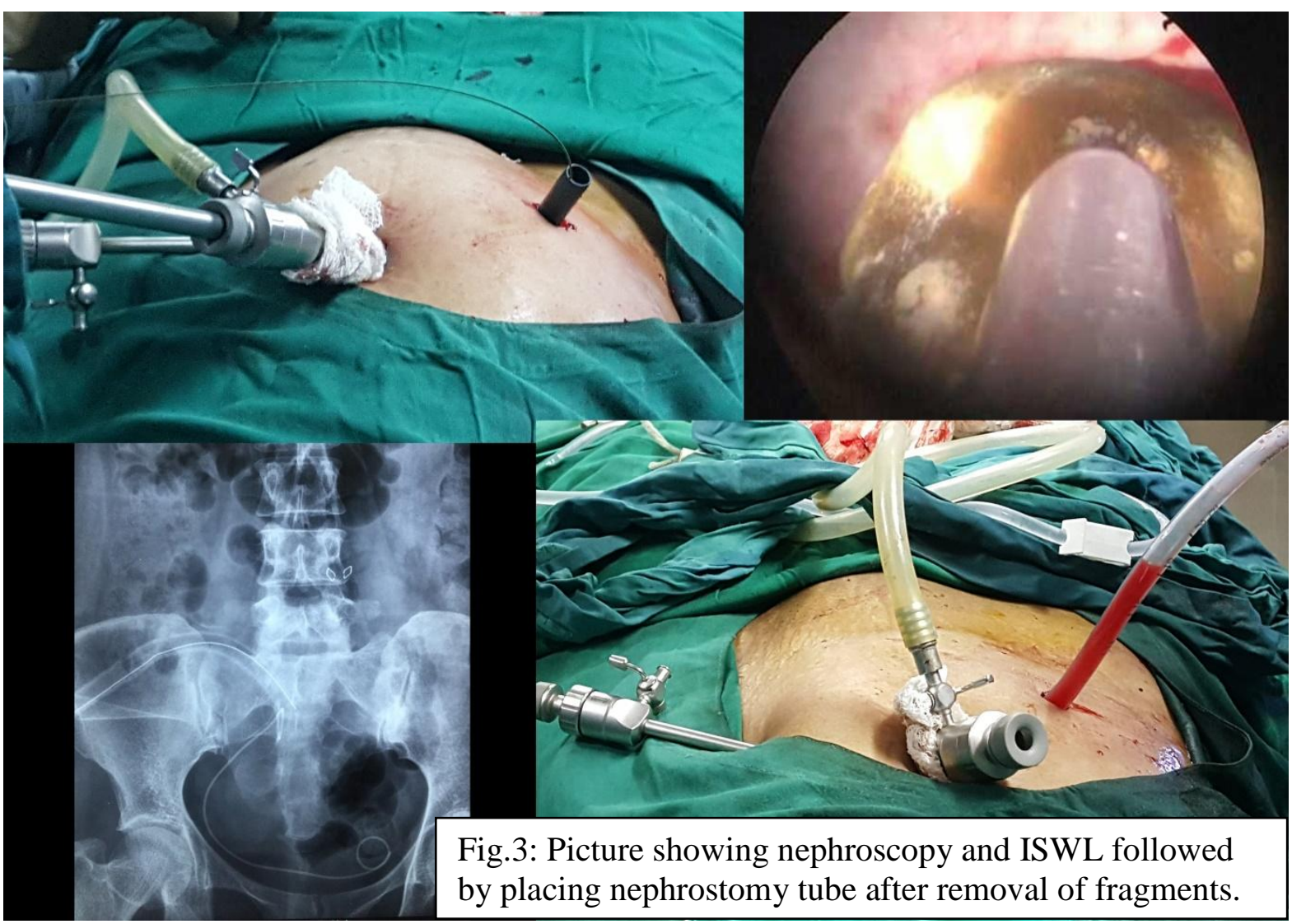

PCNL for pelvic kidney stones is also technically difficult with pt's position, and also increased risk of intra-abdominal bleeding, urinary leak, difficulty owing to abnormal orientation of pelvic kidneys with abnormal and unpredictable blood supply and surrounding bowel loops [5].

Hence such patients are candidates for Laparoscopy assisted endourological procedures.

Laparoscopy helps in mobilisation of bowel which is needed to perform puncture in transabdominal route and also helps in assisting and guiding PCNL punctures avoiding vessels and bowels [5].

Laparoscopy-guided endourological intervention permits visual exposure of the kidney, enhancing safe puncture and tract placement integral to percutaneous nephrolithotomy accomplishing stone removal by minimal invasive procedure in pelvic dystopic kidneys.

\section{References}

1. Eshghi AM, Roth JS, Smith AD. Percutaneous transperitoneal approach to a pelvic kidney for endourological removal of staghorn calculus. The Journal of Urology. 1985 Sep;134(3): 525.

2. Matlaga BR, Kim SC, Watkins SL, Kuo RL, Munch LC, Lingeman JE. Percutaneous nephrolithotomy for ectopic kidneys: over, around, or through. Urology. 2006 Mar 31;67(3):513-7.

3. Gupta NP, Mishra S, Seth A, Anand A. Percutaneous nephrolithotomy in abnormal kidneys: single-center 
experience. Urology. 2009 Apr 30; 73(4): 710-4.

4. Sohail, N., Albodour, A., \& Abdelrahman, K. (2016). Laparoscopic Assisted Transmesocolonic Percutaneous Nephrolithotripsy in Ectopic Iliac Kidney. Urology Case Reports, $7, \quad 48-50$. http://doi.org/10.1016/j.eucr.2016.04.00 5

5. D'souza N, Verma A, Rai A. Laparoscopic-assisted mini percutaneous nephrolithotomy in the ectopic pelvic kidney: Outcomes with the laser dusting technique. Urology Annals. 2016 Jan; 8(1):87.

\section{How to cite this article:}

Bhushan V. Dodia, Abhay Mahajan, Martand Patil, Sandeep Bathe and Prashant Darakh. 2017. Laparoscopy Guided PCNL in a Stone Holding Previously Operated Ectopic Pelvic Kidney. Int.J.Curr.Microbiol.App.Sci. 6(8): 975-979. doi: https://doi.org/10.20546/ijcmas.2017.608.119 